\title{
Selecting Oil Wells for Hydraulic Fracturing: A Comparison between Genetic-Fuzzy and Neuro Fuzzy Systems
}

\author{
Virgílio José Martins Ferreira Filhoㅁ, Antônio Orestes de Salvo Castro² \\ ${ }^{1}$ Production Engineering Program, Federal University of Rio de Janeiro (UFRJ), Rio de Janeiro, Brazil \\ ${ }^{2}$ Rio de Janeiro State University (UERJ), Rio de Janeiro, Brazil \\ Email: virgilio@ufri.br, orestes@petrobras.com.br
}

Received 17 May 2014; revised 20 June 2014; accepted 29 June 2014

Copyright (C) 2014 by authors and Scientific Research Publishing Inc.

This work is licensed under the Creative Commons Attribution International License (CC BY). http://creativecommons.org/licenses/by/4.0/

c) (i) Open Access

\section{Abstract}

Hydraulic fracturing is widely used to increase oil well production and to reduce formation damage. Reservoir studies and engineering analyses are carried out to select the wells for this kind of operation. As the reservoir parameters have some diffuse characteristics, Fuzzy Inference Systems (FIS) have been tested for these selection processes in the last few years. This paper compares the performance of a neuro fuzzy system and a genetic fuzzy system used for selecting wells for hydraulic fracturing, with knowledge acquired from an operational data base to set the SIF membership functions. The training data and the validation data used were the same for both systems. We concluded that, despite the genetic fuzzy system being a newer process, it obtained better results than the neuro fuzzy system. Another conclusion was that, as the genetic fuzzy system can work with constraints, the membership functions setting kept the consistency of variable linguistic values.

\section{Keywords}

Fuzzy Logic, Petroleum, Genetic Algorithms, Hydraulic Fracturing

\section{Introduction}

Stimulation operations are widespread in the oil industry to increase the productive potential of wells and hydrocarbon bearing formations. These operations act to increase productivity or injectivity of a given formation by inducing channels in reservoir rock or removal of the damage, facilitating the flow of fluids to be produced. Due to the different characteristics of each formation or project, not all wells are natural candidates to be stimu- 
lated. Even within the same oilfield wells should or should not undergo this type of operation will be found. Among the most frequent operation intervention can cite the hydraulic fracturing, acid fracturing and acid wash.

The process of selecting wells for stimulation involves the analysis of various parameters and relies heavily on technological features such as simulators and also the experience of industry experts. A poor interpretation of the parameters of the well, or a failure of the operation planning can cause serious consequences to the production of a field, and may even result in the loss of wells or serious accidents.

Thus, although stimulation operations are often practiced, the selection problem shows up still relevant and has gained increasing importance in the present scenario. Since its first commercial application in 1949, it is estimated that nearly 2.5 million fracturing operations have already been performed throughout the world and that approximately $60 \%$ of drilled wells currently suffer this kind of treatment [1]. The fracturing attracts interest not only to raise the productivity of wells, but also for providing the increase in reserves making possible the exploration of new fields - only in the United States the growth in oil reserves may have been at least $30 \%$ and in the natural gas, 90\% [2]. As important justification for maintaining high growth in research on fracturing, we can cite a field exploration of shale and tight gas. Mentioned as potential sources for the world's growing demand for natural gas [3], these reservoirs have very low permeability (less than $0.1 \mathrm{md}$ ), being thus essential to perform stimulation treatments.

The objective of this work is the presentation and analysis of a methodology to aid decision making for selection of candidate wells to stimulation using hydraulic fracturing.

Fracturing a formation successfully is still a challenge to engineers. Due to the feature of fuzzy variables involved in the selection process, professionals has become increasingly interested in intelligent systems which may serve to support decision making in many aspects of the operation, which includes everything from the selection of candidates to the wells determination of technical parameters of the intervention, in order to obtain optimum results in terms of increased productivity and reduced resource mobilization. In the last two decades, a significant activity in the area of smart computing with focus on paradigms as Artificial Neural Networks, Genetic Algorithms and Fuzzy Logic can be observed, applied to solve complex engineering problems efficiently.

Artificial Neural Networks (ANN) are on a computing paradigm based on the biological model of the human brain. Are computational techniques that have inspired neural structure and acquiring knowledge through experience, model already genetic algorithms are global optimization algorithms based on the mechanisms of natural selection and genetics, and Fuzzy Logic (Nebula) is the logic that supports modes of reasoning that are approximate rather than exact, are techniques for the treatment of qualitative information. Some studies have focused on the integration of two or more of these paradigms generating the known hybrid systems. The power of these systems relies on the fact that these techniques have complementary character, contributing their individual strength to generate a solution to the problem in question.

Currently, one of the most important areas for applying the fuzzy sets developed by Zadeh [4] is in fuzzy inference systems (FIS), which, in fact, are extensions of the classic systems with knowledge bases, but having the antecedents and conclusions of the rule "IF-THEN" made up of fuzzy statements. For the problem in question, selecting wells for hydraulic fracturing, the knowledge set is best described as a diffused composition of linguistic variables. The data used, for example the permeability of the oil formation, oil viscosity, etc., are inferred based on samples collected from drilled oil wells, therefore having uncertainty inherent to this inference process. This diffused environment for selecting wells, although involving technical and economic criteria, gives a more qualitative evaluation considering the personal characteristics of those involved in the decision making process.

The FIS for selecting wells for hydraulic fracturing works as an operational standard for this selection. As with all standards, it is fundamentally important that it is kept updated, which is not a simple task when dealing with a FIS, as it requires a specialist to acquire the knowledge and enter it into the system. Therefore, a computing system for knowledge mining and adjusting the FIS is important for keeping it operational during the period. This study presents the comparative results of using 1) a system based on genetic algorithms (GA) for updating the pertinent functions of this FIS and 2) a neural network (NN) based system for the same purpose.

Following this brief introduction, in Section 2 we review the main concepts related to FIS, neuro fuzzy systems and a genetic fuzzy systems. In Section 3, the operation of hydraulic fracturing is described as well as the key elements necessary for selection of wells for fracturing. The model developed to address the problem, the input data and results are presented in Section 4. Finally, Section 5 provides the conclusions of the study. 


\section{Fuzzy Inference System (FIS)}

\subsection{Fuzzy Sets}

Let $X$ be a space of objects, $x$, a generic element of $X$ and $A$, a crisp set such that $A \subseteq X$. Also be a collection of objects is $x \in X$, where $x$ may or may not belong to A. To define a characteristic function for each $x$ element in $X$, we can represent a set of ordered pairs $(0,1)$, which represent $x \in A$ or $x \notin A$ respectively. Differently from crisp sets, a fuzzy set expresses the membership level to a set in accordance with the interval $[0,1]$, that is, the fuzzy set $\tilde{A}$ in $X$, is a set of ordered pairs, such as $\tilde{A}=\left\{x, \mu_{A}(x) \mid x \in X\right\}$, where $\tilde{A}$ is a fuzzy set, $x$ is an element belonging to the discourse universe $X$ and $\mu_{A}(x)$ is the membership function. The membership function $\mu_{A}(x)$ defines the subjectivity of how an element may belong to a set and not the randomness of the fuzzy set. This is the fundamental difference between the fuzzy sets and the probability theory. The fuzzy sets, normally have names that correspond to the adjectives that qualify a variable (small, medium, large), which are called linguistic values, therefore the discourse universe $X$ is generally denominated as a linguistic variable.

\subsection{Fuzzy Inference System (FIS)}

The generic structure of a FIS is composed of: 1) a knowledge base (KB) which stores the available knowledge on the problem, in the form of "IF-THEN" fuzzy rules, 2) a data input fuzzification device, 3) an inference mechanism and 4) a results defuzzification device. The KB contains two different information levels: 1) the linguistic variables membership functions and 2) the production fuzzy rules. In a general form, we would have fuzzy rules of the type: IF $\boldsymbol{X}_{\mathbf{1}}$ is $\boldsymbol{A}_{\mathbf{1}}, \cdots$, and $\boldsymbol{X}_{\boldsymbol{n}}$ is $\boldsymbol{A}_{\boldsymbol{n}}$ THEN $\boldsymbol{Y}$ is $\boldsymbol{B}$, where $X_{i}$ and $Y$ are respectively inputs and outputs, and $A_{i}$ and $B$ are linguistic variables with associated fuzzy sets, therefore defining its significance. These production rules represent a fuzzy relationship between $A$ and $B$ defined in $U \times V$.

\subsubsection{Defuzzification Device}

The FIS model used for showing the selection of wells for hydraulic fracturing is the SUGENO type [5] [6]. In this type of FIS, the antecedents are composed of linguistic variables and the consequences are represented by the input variable functions. For the SUGENO type FIS, the defuzzified output is normally composed of an evaluation of $m$ rules and is obtained by the weighted total of the consequences of each rule used, $Y_{i}$, for $i=1, \cdots, m$ as described in Equation (1).

$$
\text { Defuzzified value }=\frac{\sum_{i=1}^{m} h_{i} \cdot Y_{i}}{\sum_{i=1}^{m} h_{i}}
$$

where each $h_{i}=T\left(A_{i 1}\left(x_{1}\right), \cdots, A_{i n}\left(x_{n}\right)\right)$ is the degree of adherence between the antecedent of the $i$-th rule and the current input variable of the $x_{0}=\left(x_{1}, \cdots, x_{n}\right)$ system. However, for this specific problem a new defuzzification procedure was proposed with the objective of an improved portrayal of a defuzzified value for selecting wells for hydraulic fracturing. This was due to the fact of some situations being represented by extreme values for some input variables, which would imply the summary prohibition of the well for fracturing. For example, very low variable values that show the well's mechanical conditions impede the operation and cannot be compensated by convenient values of other variables. Therefore a constraints procedure was implemented in conventional process, ensuring that determined values directly imply negative defuzzified values, in accordance with Equation (2):

$$
\text { Value }=\left\{\begin{array}{lc}
-1 & \text { if } X_{i} \leq C_{i} \forall i \\
\frac{\sum_{i=1}^{m} h_{i} \cdot Y_{i}}{\sum_{i=1}^{m} h_{i}} & \text { otherwise }
\end{array}\right.
$$

\subsubsection{FIS Membership Functions Adjustment}

The fuzzy inference model membership functions represent the input parameters containing the well/formation 
set data that may vary from one oil region to another. These parameters have different amplitudes in their values for each oil region. Therefore, although a production rule of the type if parameter $\mathbf{P}$ is high then the recommendation is $\mathbf{R}$, can be generally applied, the definition is that a high linguistic value can vary substantially depending on the oil region in which we are applying the model. Therefore the membership functions for a new environment should undergo another modeling process, similar to that which was used for establishing its linguistic values for the FIS initially modeled. To avoid such a procedure, making the model more versatile, a knowledge mining process from the field data obtained during hydraulic fracturing operations is necessary.

Two procedures for developing this capacity were compared, one involved using GA and the other based on NN, both work on the adjustment of the FIS membership functions. For implementation details of the GA adjustment model see Castro [7]. For the use of NN the ANFIS (Adaptive Neuro Fuzzy Inference System) model was chosen, as this has the capacity to emulate a SUGENO type FIS, by using radial basis neural networks.

\section{Hydraulic Fracturing}

Stimulation operations are widely used in the oil industry to increase the potential productivity of wells and hydrocarbon-bearing formations. These operations increase the permeability of the formation by inducing channels in the producing rock or by removing damage from the formation, which aids the flow of the fluids to be produced. Not all wells are natural candidates for stimulation, due to their different characteristics. Even within the same oil field and the same formation, wells will be found that should and should not undergo these operations. The selection process must be based on technical and economic criteria, which may be very difficult if applied with the necessary levels of detail. One of the main well stimulation methods used by the oil industry is hydraulic fracturing. Although it is an operation that can be very profitable, if poorly specified, designed or carried out, may even result in the loss of a production well.

Hydraulic fracturing consists of applying a pressure differential above the mechanical strength of the formation, causing it to break or fracture. Soon after pumping a volume of fluid specified high flow channels formed to propagate and injection of an agent support with pressure higher than the closing of the fractures is performed.

Due to its high rate of success and financial return, hydraulic fracturing treatments are usually performed after the drilling phase observed when the low transmission of the area of interest. After undergoing the first operation, wells that show a decline in productivity to levels below economically viable can be fractured again in order to ensure their continued operation. In large gas fields, the fracturing is one of the main operations performed and, over time; large volumes of data and significant knowledge could be acquired. When considering a hydraulic fracturing treatment, four steps must be well designed [8]:

- Selection of candidate wells.

- Design Treatment.

- Planning the operation.

- Execution of field work.

Each of these steps has similar importance, and appropriate individual attention should be paid in order to perform an efficient job. However, in this work, the focus of our attention will be devoted to the first stage, candidate selection, where engineers and operators seek ideals wells that, when fractured, can significantly increase the productivity of their field. A considerable amount of work and research has been devoted to this area using different techniques such as statistical, analytical models, simulation and intelligent computing. A literature review of this subject [9]-[12] points out that the techniques of artificial intelligence and data mining have provided great success rate when applied to selection of wells for fracturing.

\section{Selection of Wells for Hydraulic Fracturing}

The selection of candidates for stimulation is not only based on the productive potential gain of a given well, it should also ensure that this increase is sustainable, economically justifiable and not accompanied by an increase in the volume of water or gas (in the case of oil fields). In practice, most fracturing treatments are conducted in selected wells through little or no involvement of scientific foundations and engineering principles. Mostly selected candidates have low performance and the applied treatment is based on a combination of already established practices [13]. In fact, in many cases professional experience is able to solve the problem and provide satisfactory results, but this is not guaranteed and often applied treatments could have a higher yield. The selection 
of potential candidates to undergo this type of intervention does not guarantee its success. Many parameters related to the planning of the operation such as selection and design of the fracture fluid will have a direct impact on results.

The success or failure of a fracturing operation is directly tied to the quality of the candidate wells selected. To choose the best candidate to stimulation, one must take into account multiple variables. Among the most influential parameters for this type of operation can be mentioned [14]: 1) Formation permeability; 2) the level of formation damage, skin; 3) volume of oil contained in the formation; 4) formation thickness; 5) static pressure gradient; 6) well mechanical conditions; 7) oil viscosity.

A reservoir is called a low permeability one when it has a high resistance to fluid flow. In many formations, chemical and/or physical can change the properties of a reservoir rocks through geological time. Sometimes these diagenetic processes restrict the pores of the rocks thus reducing the ability of fluids to move. Rocks of low permeability are usually potential candidates for processes of stimulation by hydraulic fracturing. Extremely low permeability reservoirs cannot produce flow rates of economically viable oil, even after stimulation and thus these wells may not be good candidates.

On some occasions the permeability of the reservoir rock can be affected when the well is drilled or when the casing is seated and cemented. This effect is called formation damage. The skin or damage factor as regards the formation around the well is obstructed (or stimulated). As the main cause of obstruction we can cite the invasion of drilling fluids in the formation, altering the pores and the connection between them, and also the damage caused by firing loads on perforating. When the pores are blocked, the permeability is reduced and the reservoir flow in this region can be substantially reduced. Damage can be especially severe in naturally fractured reservoirs. To stimulate damaged reservoirs, short and high conductivity fracture is the ideal solution.

The best candidate wells are generally those with substantial volumes of hydrocarbons and need to increase their productivity index. These reservoirs have great thickness of the hydrocarbon net pay zone, medium to high pressure, in-situ stress barrier in order to contain the vertical growth of the fracture and also a zone damaged or of low permeability. Not candidates' wells generally are those with small volume of hydrocarbons in place, low pressure, and low radius of influence.

Often, the main limitations for selection of candidates are not related to technical aspects of the reservoir and fracture performance. Different aspects can cause the rejection of a range or well fracturing operation, some of these reasons are listed below [13].

\section{1) Proximity to oil-water or gas-oil contacts:}

In general, fracture zone aquifer can cause substantial damage to the performance of the well. The industry has several cases like these for obvious reasons most often are not published. There are systems capable of mitigating the effects of water penetration in areas. They act by incorporating modifiers of permeability to fracturing fluids [15] [16] or the use of proppant systems capable of maintaining open preferably the top of fracture [13] [17].

\section{2) Proximity to gas zones (for oil production):}

In oil reservoirs, fractures can easily penetrate in upper gas zones as in inferior water zones. The main difference in the case of gas zones is that few mitigation measures can be adopted.

\section{3) Limitations of completion pressures:}

Invariably fracturing requires significantly higher pressures than those experienced for completion during normal production cycles. In addition to checking the limitations of pressures in the well pipe (casing and column), particular attention should be given to how the downhole gas lift mandrels, safety valves, sliding sleeves, and apparatus for flow control. Another limitation lies in the plugs or packers. Additional pressure inside the column can result in upward vertical forces applied to packer, and the engineer must make sure that it will not be unseated or will move up. Many of these problems can be mitigated by applying pressure to the annulus, thus reducing the pressure differential between the interior of the column and the ring.

\section{4) Contraction of the column:}

Two factors may cause the reduction of column length: additional pressure and cooling caused by fracturing fluid, relatively cold. The engineer must ensure that this fact does not cause tensions above the packer or supported by the column.

\section{5) Limitations of pressure wellhead:}

Many producing wells cannot resist the necessary pressures to fracture. Thus, it is common practice to use isolation tools wellhead (treesavers) or replace the well head by a tree fracturing. Both of these options are time 
consuming and expensive operation.

5) Pipes of low quality:

Pipes for damage caused by erosion, corrosion or mechanical defects may preclude completion of a treatment because of their inability to bear the additional stresses. Damaged in the intervention column pipes can be easily replaced at an additional cost of workover, however the removal of damaged casing is much more complex and costly.

\section{7) Cementation low quality:}

Cementation is required to the isolation of a given area in order to ensure that the fracture begins in perforates and not elsewhere. Thus, one should ensure the isolation of the upper and lower area of interest, so that the operation occurs in an effective and safe way area.

\section{8) Impossibility of recovery, recycling or disposal of treatment fluids:}

The recovery of the treatment fluids is a vital part of the process, and can directly impact the execution if there is no possibility to recover it and discard it.

\section{9) Sensitivity training to the treatment fluids:}

Many formations are sensitive to water based fluids and some (especially dry gas bearing formations) to any type of fluid. There are systems that can mitigate these problems, but usually they will cause a substantial increase in treatment costs, especially if an ideal infrastructure does not exist at the location of the well.

\section{0) Isolation zone:}

Ideally, fracturing treatments should be implemented through individual perforated intervals, or open hole sections of limited length. In most cases, this isolation is relatively easy to ensure especially when the well is new, however, for existing wells, some type of intervention may be required.

\section{1) Inability to perform interventions:}

Most wells require some type of intervention before the fracturing operation. It is very rare to find a well that has a completion strategy consistent with the fracturing operation to be performed. If this adjustment operation is not possible the well cannot undergo treatment.

\section{2) Lack of infrastructure for fracturing:}

Without the existence of a basic infrastructure including tanks, water supply, recovery and disposal of fluids services, workover rigs, well testing equipment, suppliers of proppants, wire units, $\mathrm{N}_{2}$ and $\mathrm{CO}_{2}$, coiled tubing, among other is not possible a fracturing operation. The costs associated with this provision depending on the location of the well and the market can make the operation unviable.

\section{3) Well location:}

Some wells are more accessible and therefore cheaper to treat than others. Offshore or difficult to access onshore wells multiply the costs of an operation.

\section{4) High pressure and temperature wells:}

In such cases, special care must be taken and more resistant equipment should be used. It should also be aware of the existence or availability fluid or proppant material capable of withstanding these conditions.

\section{Model for Selecting Oil Wells for Hydraulic Fracturing}

\subsection{Model Input and Output Variables}

For feeding the model, various factors must be considered in the selection of wells and formations for hydraulic fracturing. As pointed in previous section, the seven most important factors in the selection process were identified as being the following: 1) the level of formation damage, skin; 2) formation thickness; 3) volume of oil contained in the formation; 4) static pressure gradient; 5) well mechanical conditions; 6) formation permeability and 7) oil viscosity.

To identify the wells that are candidates for hydraulic fracturing operations, a classification was made in line with a possible diagnostic from a specialist who might analyze the problem. This evaluation consists of classifying the wells into four groups in accordance with their suitability for hydraulic fracturing: 1) excellent candidate; 2) good candidate; 3) possible candidate and 4) non candidate. The FIS is able, based on the seven characteristics of an oil well, to classify it in one of the above four classes. Firstly the wells are placed in ascending order of suitability, with the model firstly generating negative values for the non-candidate wells, values between 0 and 0.5 for possible candidates, between 0.5 and 0.75 for good candidates and above 0.75 for excellent candidates. 


\subsection{Data for Knowledge Extraction and Validation}

For adjusting the membership functions of the SIF, a survey was conducted in the corporate database used to store data from producing wells. 110 operations performed in different fracturing oil wells have been selected. The data collected were the values of the 7 input parameters used in the FIS and the value of the increased production resulting from the fracturing operation. This gain is to define the order of the wells obeying the criterion of increasing order of suitability. To the data of these wells, data from another 50 wells was added that were nonfractured, as they didn't have ideal characteristics for the operation. In these wells, the increase in production was entered as zero. This second set of data was aimed at increasing knowledge in respect to wells in which the action is not recommended. This set of data, a total of 100 wells (70 fractured and 30 non-fractured) was used to define the membership functions with the use of GAs. Another 60 wells (40 fractured and 20 non-fractured) were separated for testing and validating the generated model.

\subsection{Genetic Fuzzy Systems}

The genetic fuzzy systems adjust the knowledge contained in a FIS by minimizing output errors from the adjusted FIS and the results from a training set used for comparison. This process includes the creation of genetic material, generation of initial solutions, evaluation of errors by a fitness function, which will be used in the selection process to determine the most suitable individuals and which will have the greatest chance of reproduction. To this process is added an eventual mutation process to generate new individuals, increasing the search space and obliging the procedure to move from local minimums. Therefore this is an iterative process which will end when the error reaches an acceptable value. It is possible to include constraints in this process, within the fitness function, which proved to be extremely useful both for reducing the convergence time and for maintaining the linguistic significance of the fuzzy set membership functions. For implementation details of the GA adjustment model see Castro [7].

\subsection{Genetic Fuzzy System Results}

Table 1 shows the results extracted after running the model six times with GAs. In the first column, we have a description of the input variable, followed by its value before the use of the GAs to adjust the membership functions. The columns numbered one to six show the values obtained from each of the model's six runs. The calculated average values, shown in the next column, where used to redesign the membership functions. The standard deviation and the variation coefficient show the dispersion between the values obtained from each run. We can see that the value of point 3 for permeability, the value of point 6 for oil volume and the value of point 3 for formation thickness have an elevated variation coefficient, showing no convergence.

Figure 1 shows the membership functions before the adjustment process by genetic algorithms (fine line) and after adjustment (darker line). The numbers beside the linguistic values make the relationship to Table 1 , so that its values can be found in the figures. Here we can see more clearly that the permeability linguistic variable "high" value, the volume of oil "very high" value and the thickness "very high" value, as demonstrated by the variation coefficient, really showed no convergence. This is due to the non existence of values for these parameters within these intervals in the wells used for training. A modification of the model was made for this event, so that the adjusted values that didn't show convergence, don't substitute the former values of the FIS membership functions. However, the majority of adjustments shown by the model was very adequate and had convergence. The set of 60 wells (40 fractured and 20 non-fractured), separated for validating the model, had an error considered very low, as of the 40 fractured wells, 13 were out of their expected position, not generating an individual position error greater than seven units. For the set of 20 non-fractured wells, only one had a suitability value greater than zero (only negative suitability values were expected for these wells), however this one well had a value very close to zero [0.0023].

\subsection{The ANFIS Model}

The ANFIS procedure used, automatically adjusted a SUGENO type FIS. It was used for applying a hybrid training strategy, formed by combining the minimum squared and descending gradient methods, generating an output described by the consequences weighted linear combination. There is no formal theory for determining the topology of the network for a given application, therefore the original FIS was used, which was developed 
Table 1. Results obtained with a genetic fuzzy system.

\begin{tabular}{|c|c|c|c|c|c|c|c|c|c|c|c|}
\hline \multirow{2}{*}{$\begin{array}{l}\text { Linguistic } \\
\text { variable }\end{array}$} & \multirow{2}{*}{$\begin{array}{c}\text { Non- } \\
\text { adjusted }\end{array}$} & \multirow{2}{*}{ Point } & \multicolumn{6}{|c|}{ Values obtained from running the model } & \multirow{2}{*}{ Average } & \multirow{2}{*}{$\begin{array}{l}\text { Standard } \\
\text { deviation }\end{array}$} & \multirow{2}{*}{$\begin{array}{c}\text { Variation } \\
\text { coef. }\end{array}$} \\
\hline & & & 1 & 2 & 3 & 4 & 5 & 6 & & & \\
\hline \multirow{3}{*}{$\begin{array}{l}\text { Skin (form. } \\
\text { damage) }\end{array}$} & 0 & 1 & -1.7 & -1.74 & -1.71 & -1.76 & -1.74 & -1.79 & -1.740 & 0.0300 & 0.01724 \\
\hline & 2.5 & 2 & 3.15 & 3.21 & 3.17 & 3.18 & 3.18 & 3.19 & 3.180 & 0.0183 & 0.00574 \\
\hline & 6 & 3 & 8.76 & 8.59 & 8.61 & 8.52 & 8.54 & 8.5 & 8.587 & 0.0863 & 0.01006 \\
\hline \multirow{3}{*}{$\begin{array}{l}\text { Pressure } \\
\text { gradient }\end{array}$} & 0.25 & 1 & 0.231 & 0.203 & 0.207 & 0.214 & 0.214 & 0.205 & 0.212 & 0.0093 & 0.04398 \\
\hline & 0.50 & 2 & 0.418 & 0.42 & 0.431 & 0.411 & 0.413 & 0.427 & 0.420 & 0.0071 & 0.01695 \\
\hline & 0.65 & 3 & 0.589 & 0.595 & 0.592 & 0.597 & 0.591 & 0.594 & 0.593 & 0.0026 & 0.00446 \\
\hline \multirow{3}{*}{$\begin{array}{l}\text { Formation } \\
\text { permeability }\end{array}$} & 400 & 1 & 525 & 532 & 517 & 529 & 543 & 539 & 530.833 & 8.6104 & 0.01622 \\
\hline & 1000 & 2 & 1232 & 1259 & 1274 & 1232 & 1295 & 1242 & 1255.667 & 23.0988 & 0.01840 \\
\hline & 2500 & 3 & 2711 & 3812 & 4932 & 5083 & 5229 & 6536 & 4717.167 & 1196.812 & 0.25371 \\
\hline \multirow{4}{*}{$\begin{array}{c}\text { Mechanical } \\
\text { conditions }\end{array}$} & 0.35 & 1 & 0.354 & 0.361 & 0.359 & 0.341 & 0.349 & 0.348 & 0.352 & 0.0068 & 0.01941 \\
\hline & 0.52 & 2 & 0.551 & 0.498 & 0.502 & 0.509 & 0.511 & 0.499 & 0.512 & 0.0182 & 0.03564 \\
\hline & 0.75 & 3 & 0.712 & 0.719 & 0.727 & 0.718 & 0.721 & 0.741 & 0.723 & 0.0092 & 0.01270 \\
\hline & 0.90 & 4 & 0.919 & 0.923 & 0.917 & 0.921 & 0.965 & 0.916 & 0.927 & 0.0172 & 0.01859 \\
\hline \multirow{4}{*}{ Oil viscosity } & 20 & 1 & 0.02 & 0.019 & 0.021 & 0.024 & 0.021 & 0.023 & 0.021 & 0.0017 & 0.07967 \\
\hline & 100 & 2 & 113 & 109 & 103 & 121 & 116 & 109 & 111.833 & 5.7276 & 0.05122 \\
\hline & 370 & 3 & 391 & 396 & 389 & 401 & 394 & 390 & 393.500 & 4.1130 & 0.01045 \\
\hline & 700 & 4 & 721 & 697 & 718 & 703 & 709 & 711 & 709.833 & 8.2141 & 0.01157 \\
\hline \multirow{6}{*}{ Oil volume } & 50 & 1 & 21.4 & 22.6 & 21.3 & 19.7 & 17.9 & 19.1 & 20.333 & 1.5839 & 0.07790 \\
\hline & 550 & 2 & 400 & 398 & 414 & 429 & 419 & 413 & 412.167 & 10.6680 & 0.02588 \\
\hline & 630 & 3 & 605 & 619 & 612 & 607 & 601 & 618 & 610.333 & 6.6249 & 0.01085 \\
\hline & 1100 & 4 & 1024 & 1043 & 1002 & 1007 & 1029 & 1036 & 1023.500 & 14.7281 & 0.01439 \\
\hline & 1250 & 5 & 1196 & 1251 & 1163 & 1239 & 1236 & 1243 & 1221.333 & 31.4148 & 0.02572 \\
\hline & 1750 & 6 & 1201 & 1958 & 1690 & 1342 & 1823 & 2196 & 1701.667 & 342.7724 & 0.20143 \\
\hline \multirow{3}{*}{$\begin{array}{l}\text { Formation } \\
\text { thickness }\end{array}$} & 10 & 1 & 12.9 & 14 & 10.4 & 13.7 & 13.6 & 12.3 & 12.817 & 1.2185 & 0.09507 \\
\hline & 23 & 2 & 21.7 & 22.5 & 18.6 & 19.9 & 20.1 & 17.8 & 20.100 & 1.6279 & 0.08099 \\
\hline & 35 & 3 & 31.6 & 39.7 & 85.9 & 48.7 & 62.7 & 43.9 & 52.083 & 17.8307 & 0.34235 \\
\hline
\end{tabular}

for modeling from the knowledge of specialists. This factor was also important as it allowed comparison between the performance of the ANFIS and the procedures using GAs, developed in this work, as this basic model was also used in the membership function adjustment with GAs procedure. The implementation was carried out at MATLAB using the TOOLBOX ANFISEDIT commercial application and showed rapid convergence if compared to the procedure with GAs and with a relatively small number of iterations. The error had rapid reduction, demonstrating the efficiency of the methodology. This study took, on average, 110 minutes, with a "Celeron 600" CPU, until the error reached values of less than $1 \%$. This time was considered short if compared to traditional procedures that use neural networks and even if compared to the competing GA procedure, which took $190 \mathrm{mi}-$ nutes with the same CPU. 

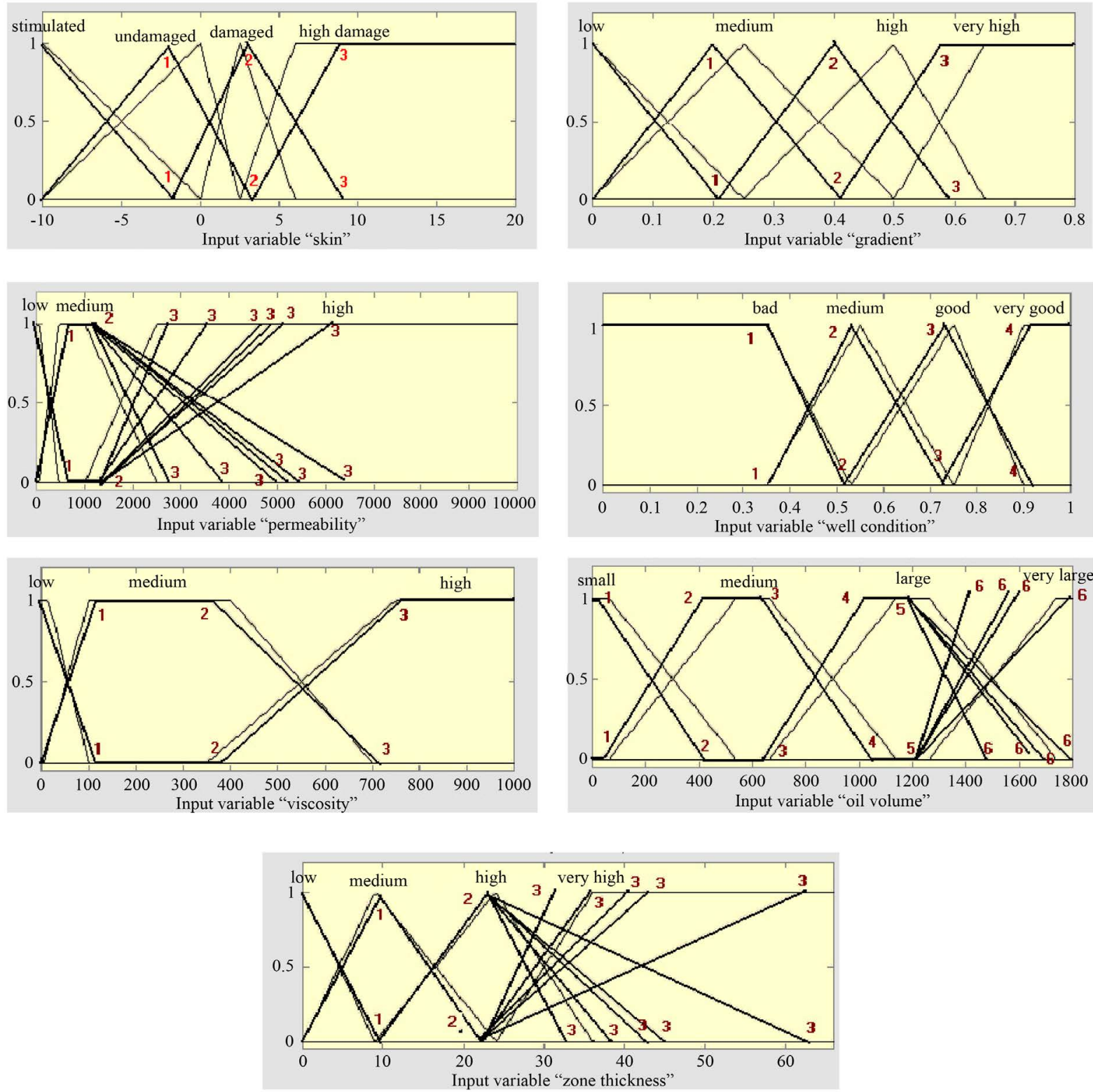

Figure 1. Adjusted membership functions table by the genetic fuzzy system.

\subsubsection{ANFIS Results-Input Variables Membership Functions}

The input variables membership functions were altered considerably by the ANFIS procedure. In this procedure there are no links to the possibilities of altering the membership functions, therefore these alterations occurred in a manner that the fuzzy sets, in most cases, lost connection with their linguistic value. Table 2 shows, for each of the model's input variables, the values before and after adjustment of the four points which form the four vertices of the trapezoidal fuzzy number of each linguistic value. We can see that points 3 and 4 , which represent the upper vertices of the fuzzy numbers trapezoid after adjustment, are equal. This occurred as it was specified in the MATLAB package which implements the ANFIS, as these functions would be represented by triangular numbers.

Figure 2 shows the input variables fuzzy sets, before and after adjustment in continuous and plotted lines respectively. Various interesting aspects can be seen, among these are highlighted: 1) The linguistic variable gradient high fuzzy set extends across almost the whole range of values, showing little membership at the intersection with the low set, including the entire medium set and with significant membership at the intersection with 
Table 2. Results obtained with the neuro fuzzy system.

\begin{tabular}{|c|c|c|c|c|c|c|c|c|c|}
\hline \multirow{2}{*}{ Variable } & \multirow{2}{*}{ Linguistic value } & \multicolumn{4}{|c|}{ Before adjustment } & \multicolumn{4}{|c|}{ After adjustment } \\
\hline & & 1 & 2 & 3 & 4 & 1 & 2 & 3 & 4 \\
\hline \multirow{4}{*}{ Gradient } & Low & 0 & 0 & 0 & 0.25 & 0 & 0 & 0 & 0.18 \\
\hline & Medium & 0 & 0.25 & 0.25 & 0.5 & 0.14 & 0.26 & 0.26 & 0.52 \\
\hline & High & 0.25 & 0.5 & 0.5 & 0.65 & 0 & 0.47 & 0.47 & 0.75 \\
\hline & Very high & 0.5 & 0.65 & 1.02 & 1.5 & 0.52 & 0.91 & 0.91 & 1.47 \\
\hline \multirow{4}{*}{ Skin } & Stimulated & -10 & -10 & -10 & 0 & -10 & -10 & -10 & -2.4 \\
\hline & Undamaged & -10 & 0 & 0 & 2.5 & -10 & -6.3 & 6.3 & 4.2 \\
\hline & Damaged & 0 & 2.5 & 2.5 & 7.5 & -0.5 & 4.4 & 4.4 & 13.9 \\
\hline & High damage & 2.5 & 7.5 & 20 & 20 & 4.4 & 17.8 & 17.8 & 20 \\
\hline \multirow{4}{*}{$\begin{array}{c}\text { Mechanical } \\
\text { conditions }\end{array}$} & Bad & 0 & 0 & 0.35 & 0.5 & 0 & 0 & 0 & 0.06 \\
\hline & Medium & 0.35 & 0.5 & 0.5 & 0.75 & 0 & 0.07 & 0.07 & 0.28 \\
\hline & Good & 0.5 & 0.75 & 0.75 & 0.9 & 0.1 & 0.45 & 0.45 & 0.84 \\
\hline & Very good & 0.75 & 0.9 & 1 & 1 & 0.31 & 0.9 & 0.9 & 1 \\
\hline \multirow{3}{*}{ Permeability } & Low & 0 & 0 & 0 & 70 & 0 & 90 & 90 & 280 \\
\hline & Medium & 0 & 70 & 150 & 250 & 0 & 295 & 295 & 563 \\
\hline & High & 100 & 250 & 10000 & 10000 & 284 & 9564 & 9564 & 12000 \\
\hline \multirow{3}{*}{ Viscosity } & Low & 0 & 0 & 40 & 100 & 0 & 121 & 121 & 312 \\
\hline & Medium & 0 & 100 & 380 & 700 & 0 & 507 & 507 & 1000 \\
\hline & High & 380 & 700 & 1000 & 1000 & 486 & 769 & 769 & 1000 \\
\hline \multirow{4}{*}{ Thickness } & Low & 0 & 0 & 5 & 10 & 0 & 0 & 0 & 9 \\
\hline & Medium & 5 & 10 & 10 & 25 & 2 & 14 & 14 & 31 \\
\hline & High & 10 & 25 & 25 & 35 & 12 & 26 & 26 & 40 \\
\hline & Very high & 25 & 35 & 100 & 100 & 32 & 62 & 62 & 100 \\
\hline
\end{tabular}

the very high set. This description of the behavior of this set, is totally different from the idea that a specialist had about a high linguistic value gradient; 2) The same occurs with the medium set of the viscosity variable, which includes the whole range of values; 3 ) This same fact can also be seen for the medium set of the oil volume variable.

\subsubsection{ANFIS Results-Output Variable}

It was necessary to increase the quantity of the output linguistic variable values (recommendation), in a manner that it would have the same quantity of values as the quantity of rules shown by the FIS, increasing from 4 to 29 . As the additional linguistic values refer to the four original linguistic values, they were initialized as the corresponding numerical values, that is, the additional unadvisable skin and gradient values, as they refer to the original unadvisable value, were initialized as -1 . At the end of the training process an interesting result was verified. The additional values converge at numbers totally unconnected to their original values, both in respect to the quantitative value and the relative value concerning the signal. Table 3 shows the linguistic values of the fuzzy sets that represent the output variable, before and after adjustment using the ANFIS. We can see, as observed in the input data, the complete loss of significance of these values with the premise used in the modeling, which identified the situations not recommended for hydraulic fracturing as negatives. Consequently any individual 

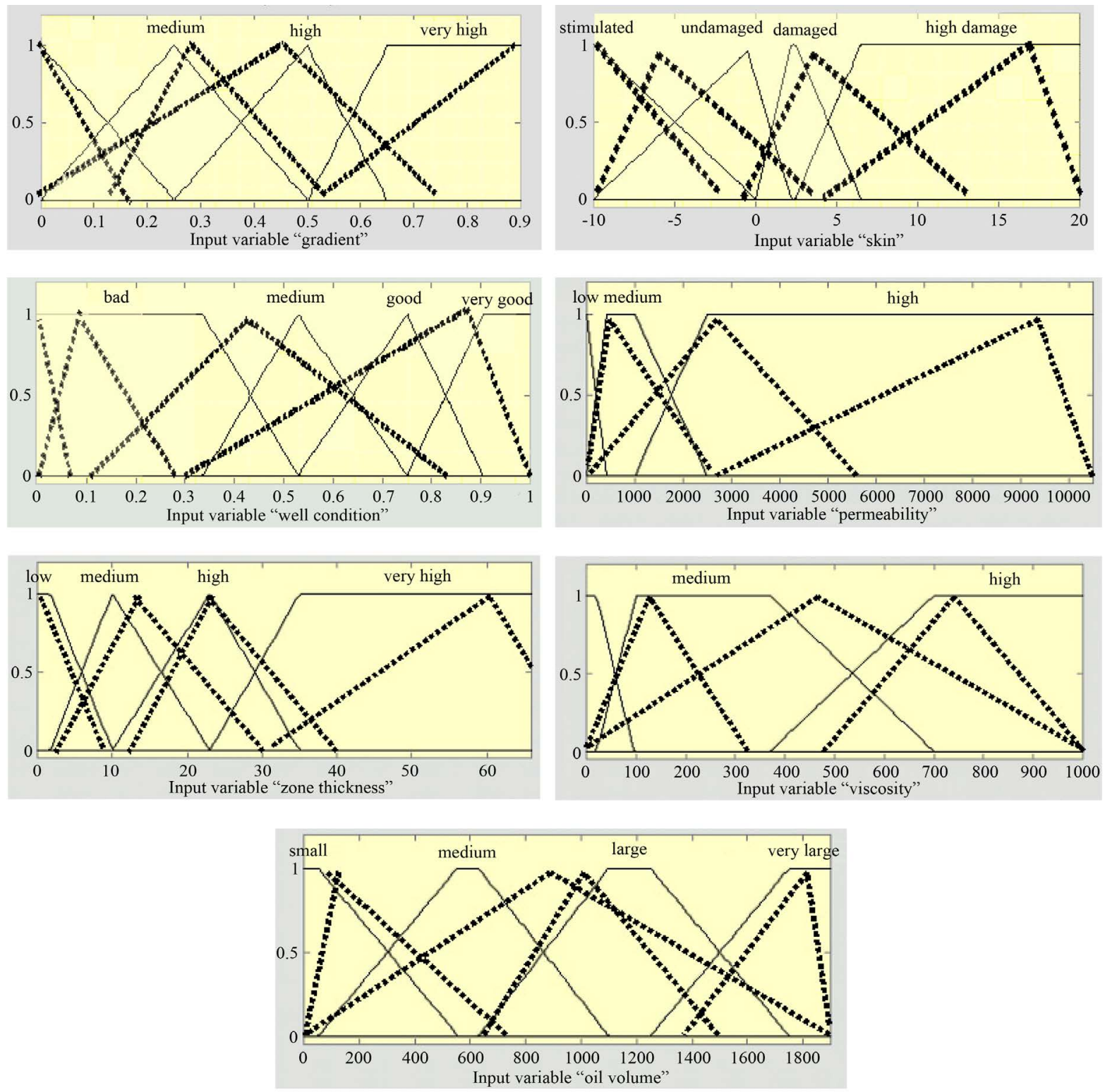

Figure 2. Adjusted membership functions table by the neuro fuzzy system.

qualitative analysis of the FIS parameters after adjustment, is not possible, as they only have a mathematical correlation to the input parameters. Therefore, when submitting a well to the FIS, this mathematical correlation will produce satisfactory results, but must function as a black box without attempting to understand the individual logic and each numerical value corresponding to the linguistic values.

\section{Comparison between the Ordering by the GA Model and the ANFIS Model}

After adjustment of the membership functions by the ANFIS procedure, the set composed of 60 validation wells (40 fractured and 20 non-fractured) was input to the FIS adjusted by the ANFIS, in the same manner as when adjusted using the GAs. Table 4 shows the ordering made by the FIS adjusted by the GAs and then, below, the ordering obtained by the FIS after adjustment using the ANFIS procedure. In both cases, the error was calculated considering only the difference in the ordering expected and that obtained. For the set of 20 non-fractured wells, only negative suitability values are expected.

We can see that the well quantitative that does not obey the expected ordering after adjustment by the ANFIS 
Table 3. Linguistic values of the output variable, before and after adjustment using the ANFIS.

\begin{tabular}{|c|c|c|c|c|}
\hline \multicolumn{3}{|c|}{ BEFORE adjustment } & \multicolumn{2}{|c|}{ AFTER adjustment } \\
\hline & istic value & Numeric value & Numeric value & Linguistic value \\
\hline 1 & Unadvisable & -1 & -47.49 & Skin unadvisable \\
\hline 2 & Possible & 0.25 & -46.85 & Skin possible \\
\hline 3 & Good & 0.5 & -47.02 & Skin good \\
\hline 4 & Excellent & 1 & -46.09 & Skin excellent \\
\hline 1 & Unadvisable & -1 & 0 & Gradient unadvisable \\
\hline 2 & Possible & 0.25 & 0 & Gradient possible \\
\hline 3 & Good & 0.5 & 114.5 & Gradient good \\
\hline 4 & Excellent & 1 & 113.3 & Gradient excellent \\
\hline 1 & Unadvisable & -1 & 2.239 & Conditions unadvisable \\
\hline 2 & Possible & 0.25 & 2.153 & Conditions possible \\
\hline 3 & Good & 0.5 & 0.917 & Conditions good \\
\hline 4 & Excellent & 1 & 1.107 & Conditions excellent \\
\hline 2 & Possible & 0.25 & -35.81 & Thickness possible \\
\hline 3 & Good & 0.5 & -35.54 & Thickness good \\
\hline 4 & Excellent & 1 & 0 & Thickness excellent \\
\hline 2 & Good & 0.5 & -34.9 & Thickness unadvisable \\
\hline 1 & Unadvisable & -1 & 0 & Perm x Visc. unadvisable \\
\hline 1 & Unadvisable & -1 & -107 & Perm x Visc. unadvisable \\
\hline 2 & Possible & 0.25 & -171.7 & Perm x Visc. possible \\
\hline 2 & Possible & 0.25 & -2.33 & Perm x Visc. possible \\
\hline 3 & Good & 0.5 & -3.02 & Perm x Visc. good \\
\hline 3 & Good & 0.5 & 4.584 & Perm x Visc. good \\
\hline 4 & Excellent & 1 & -3.271 & Perm x Visc. excellent \\
\hline 4 & Excellent & 1 & 3.178 & Perm x Visc. excellent \\
\hline 3 & Good & 0.5 & 7.298 & Perm x Visc. good \\
\hline 1 & Unadvisable & -1 & -36.76 & Volume unadvisable \\
\hline 2 & Possible & 0.25 & -35.58 & Volume possible \\
\hline 3 & Good & 0.5 & -34.5 & Volume good \\
\hline 4 & Excellent & 1 & 0 & Volume excellent \\
\hline
\end{tabular}

procedure (18 wells), was greater than when adjusted by the GAs (13 wells), however, the total error was less. While the error made by the FIS adjusted by the GAs was 40 positions, the error of the FIS adjusted by the ANFIS procedure was 35 positions. This means that, although the FIS adjusted by the GAs had placed less wells out of the expected position, when it did, they were placed further from these positions than the FIS adjusted by the ANFIS procedure. This implies a similar performance for the two procedures for this subset (40 fractured wells).

Table 5 shows the FIS output values for the non-fractured wells of the validation set. It shows that, although a great number of wells had positive values, these were very close to zero, except for well 13 which had an output 
Table 4. Comparison between ordering errors: genetic fuzzy system-neuro fuzzy system.

\begin{tabular}{cccccccccccccccccccccc}
\hline Well-expected position & $\mathbf{1}$ & $\mathbf{2}$ & $\mathbf{3}$ & $\mathbf{4}$ & $\mathbf{5}$ & $\mathbf{6}$ & $\mathbf{7}$ & $\mathbf{8}$ & $\mathbf{9}$ & $\mathbf{1 0}$ & $\mathbf{1 1}$ & $\mathbf{1 2}$ & $\mathbf{1 3}$ & $\mathbf{1 4}$ & $\mathbf{1 5}$ & $\mathbf{1 6}$ & $\mathbf{1 7}$ & $\mathbf{1 8}$ & $\mathbf{1 9}$ & $\mathbf{2 0}$ \\
\hline GA model position & 1 & 3 & 7 & 2 & 5 & 6 & 4 & 8 & 9 & 10 & 11 & 16 & 13 & 12 & 15 & 14 & 17 & 18 & 19 & 24 \\
Error & 0 & 1 & 4 & 2 & 0 & 0 & 3 & 0 & 0 & 0 & 0 & 4 & 0 & 2 & 0 & 2 & 0 & 0 & 0 & 4 \\
ANFIS model position & 2 & 6 & 1 & 3 & 5 & 4 & 7 & 8 & 9 & 11 & 10 & 12 & 13 & 14 & 15 & 16 & 17 & 20 & 19 & 21 \\
error & 1 & 4 & 2 & 1 & 0 & 2 & 0 & 0 & 0 & 1 & 1 & 0 & 0 & 0 & 0 & 0 & 0 & 2 & 0 & 1 \\
\hline Well-expected position & $\mathbf{2 1}$ & $\mathbf{2 2}$ & $\mathbf{2 3}$ & $\mathbf{2 4}$ & $\mathbf{2 5}$ & $\mathbf{2 6}$ & $\mathbf{2 7}$ & $\mathbf{2 8}$ & $\mathbf{2 9}$ & $\mathbf{3 0}$ & $\mathbf{3 1}$ & $\mathbf{3 2}$ & $\mathbf{3 3}$ & $\mathbf{3 4}$ & $\mathbf{3 5}$ & $\mathbf{3 6}$ & $\mathbf{3 7}$ & $\mathbf{3 8}$ & $\mathbf{3 9}$ & $\mathbf{4 0}$ \\
\hline GA model position & 21 & 20 & 23 & 22 & 25 & 26 & 27 & 28 & 29 & 30 & 31 & 32 & 37 & 34 & 35 & 36 & 40 & 38 & 39 & 33 \\
Error & 0 & 2 & 0 & 2 & 0 & 0 & 0 & 0 & 0 & 0 & 0 & 0 & 4 & 0 & 0 & 0 & 3 & 0 & 0 & 7 \\
ANFIS model position & 18 & 27 & 24 & 22 & 24 & 26 & 23 & 28 & 29 & 30 & 31 & 32 & 34 & 35 & 33 & 36 & 37 & 38 & 39 & 40 \\
\hline Error & 3 & 5 & 1 & 2 & 1 & 0 & 4 & 0 & 0 & 0 & 0 & 0 & 1 & 1 & 2 & 0 & 0 & 0 & 0 & 0 \\
\hline
\end{tabular}

Table 5. Output values for wells not recommended-neuro fuzzy system.

\begin{tabular}{cccc}
\hline Well & FIS output & Well & FIS output \\
\hline 1 & -0.0026 & 11 & -0.6115 \\
2 & 0.0077 & 12 & 0.0019 \\
3 & -0.0040 & 13 & 0.1389 \\
4 & -0.4379 & 14 & -0.0002 \\
5 & -0.0662 & 15 & -0.4225 \\
6 & 0.0040 & 16 & -0.0075 \\
7 & -0.0387 & 17 & -0.1984 \\
8 & 0.0060 & 18 & -0.0088 \\
9 & -0.1062 & 19 & 0.0034 \\
10 & 0.0021 & 20 & -0.3079 \\
\hline
\end{tabular}

value of 0.1389 , which is significantly greater than 0 for the analysis in question, placing it definitely in the category of possible wells for fracturing. Deeper analysis of this well showed that it had serious mechanical problems that impeded its fracturing and that the output value given by the FIS adjusted by the GAs had been -1 , originating from the defuzzification with constraints procedure, that is programmed to show a specific value in a determined situation (see Equation (2)), in this case the mechanical condition of the well with a BAD linguistic value. Another two wells were also in this situation, well 11, which had an output value of -0.6115 and well 3 , which had an output value of -0.0040 . In these two cases, other input variable conditions were not sufficiently favorable to compensate for the mechanical problem, however it was evident that the defuzzification with constraints procedure was important and should be input to the FIS after the adjustment by the ANFIS procedure.

\section{Conclusions}

In the case analyzed, despite both the neuro fuzzy system and the genetic fuzzy system having showed good results, in respect of the FIS membership functions adjustment, modeled to select oil wells for hydraulic fracturing, some significant advantages were seen in the genetic fuzzy system, although the neuro fuzzy system already had a consolidated technique, for example:

- In the ANFIS, due to an absence of constraints, some FIS membership functions lost the connection with the specialists' logic. In fact, the restrictive process allowed by the GAs was very useful, mainly for maintaining the geometric consistency of the fuzzy set, which was even broadened during the experiment, with the in- 
corporation of additional constraints, which effectively forced the maintenance of the fuzzy variables' linguistic logic. Despite not having been tested, it is believed from experience acquired during this work, that without this set of constraints the membership functions' adjustment procedure, using GAs, would have a performance similar to the ANFIS, i.e. obtaining good results, but losing the linguistic significance of some membership functions.

- Another characteristic of implementing the ANFIS is that it wasn't possible to use the defuzzification with constraints procedure, developed specifically for this type of FIS. For the methodology using GAs, the FIS itself was part of the fitness function; therefore there was no impediment in respect to this defuzzification process. In the ANFIS, there is no way to emulate in the neural network the dufuzzification with constraints procedure. Therefore, the flexibility offered by the GAs allowed the defuzzification with constraints process, important for system validation, to be maintained during adjustment.

- A significant programming effort is necessary for implementing the adjustment with GAs procedure, mainly for describing the fitness function, however this effort is worthwhile and, as this function is the adjusted FIS itself, any type of FIS may be used, such as, for example MANDANI type systems. With the mass use of this procedure and the consequent implementation of commercial applications, this effort would decrease significantly. However, if the reduction of this effort signifies the simplification of the process for including constraints (difficult to implement in an automatic manner) the method's potential will be lost.

- It was necessary to alter the number of membership functions in the output fuzzy set in the adjustment made by the ANFIS, so that it would remain with a number of functions equal to the number of rules. Although this modification doesn't alter the performance of the system, it adds a large quantity of redundant fuzzy sets.

- Alterations were made to the value of the parameters, which define the membership functions of the output fuzzy set, for the adjustment by the ANFIS. Therefore, as well as an increase to its linguistic variables' quantitative, its standard values were altered. This resulted in a large discrepancy in the significance of each of these variables, as previously the four variables (excellent, good, possible and unadvisable) had decreasing values from 1 (excellent) to -1 (unadvisable). After adjustment these values lost the correlation with this logic, as, for example excellent in the Skin rule obtained a negative value (-46.09), when originally it was positive (1). Therefore, in the FIS knowledge base, we can state that it has knowledge, but could not be validated by business logic, being only a set of mathematical relationships which have satisfactory output results.

\section{References}

[1] Mohaghegh, S., Reeves, S. and Hill, D. (2000) Development of an Intelligent Systems Approach for Restimulation Candidate. Proceedings of SPE/CERI Gas Technology Symposium, Calgary, 3-5 April 2000, 1-11. http://dx.doi.org/10.2118/59767-MS

[2] Marongiu-Porcu, M., Economides, M.J. and Holditch, S.A. (2013) Economic and Physical Optimization of Hydraulic Fracturing. Journal of Natural Gas Science and Engineering, 14, 91-107. http://dx.doi.org/10.1016/j.jngse.2013.06.001

[3] Krauss, C. (2009) New Way to Tap Gas May Expand Global Supplies. New York Times.

[4] Zadeh, L. (1965) Fuzzy Sets. Information and Control, 8, 338-353. http://dx.doi.org/10.1016/S0019-9958(65)90241-X

[5] Takagi, T. and Sugeno, M. (1985) Fuzzy Identification of Systems and Its Applications to Modeling and Control. IEEE Transactions on Systems, Man, and Cybernetics, SMC-15, 116-132. http://dx.doi.org/10.1109/TSMC.1985.6313399

[6] Sugeno, M., Kang, T. and Kang, G. (1988) Structure Identification of Fuzzy Model. Fuzzy Sets and Systems, 28, 15-33. http://dx.doi.org/10.1016/0165-0114(88)90113-3

[7] de Salvo Castro, A.O. and Ferreira Filho, V.J.M. (2005) Selecting Oil Wells for Hydraulic Fracturing: A Comparison between Genetic-Fuzzy and Neuro Fuzzy Systems. (Seleção de Poços de Petróleo para Fraturamento Hidráulico : Uma Comparação entre Sistemas Genético-Fuzzy e Neuro-Fuzzy—In Portuguese). Simpósio Brasileiro de Pesquisa Operacional, SOBRAPO, Gramado.

[8] Popa, A. and Wood, W. (2011) Application of Case-Based Reasoning for Well Fracturing Planning and Execution. Journal of Natural Gas Science and Engineering, 3, 687-696. http://dx.doi.org/10.1016/j.jngse.2011.07.013

[9] Shelley, R. and Grieser, W. (1999) Artificial Neural Network Enhanced Completions Improve Well Economics. Proceedings of SPE Hydrocarbon Economics and Evaluation Symposium (Society of Petroleum Engineers), Dallas, 21-23 March 1999, 1-11. http://dx.doi.org/10.2118/52959-MS

[10] Reeves, S.R. (1998) Fracture-Stimulation Technology for Gas-Storage Wells. Journal of Petroleum Technology, 50, 


\section{1-67. http://dx.doi.org/10.2118/39417-JPT}

[11] Reeves, S.R., Bastian, P.A., Spivey, J.P., Flumerfelt, R.W., Mohaghegh, S. and Koperna, G.J. (2000) Benchmarking of Restimulation Candidate Selection Techniques in Layered, Tight Gas Sand Formations Using Reservoir Simulation. Proceedings of SPE Annual Technical Conference and Exhibition (Society of Petroleum Engineers), Dallas, 1-4 October 2000, 1-16. http://dx.doi.org/10.2118/63096-MS

[12] Mohaghegh, S., Platon, V. and Ameri, S. (2001) Intelligent Systems Application in Candidate Selection and Treatment of Gas Storage Wells. Journal of Petroleum Science and Engineering, 31, 125-133. http://dx.doi.org/10.1016/S0920-4105(01)00125-5

[13] Martin, A. and Economides, M. (2010) Best Practices for Candidate Selection, Design and Evaluation of Hydraulic Fracture Treatments. Proceedings of SPE Production and Operations Conference and Exhibition (Tunnis: Society of Petroleum Engineers), Tunis, 8-10 June 2010, 1-13. http://dx.doi.org/10.2118/135669-MS

[14] de S. Castro, A.O. (2005) Selecting Oil Wells for Hydraulic Fracturing: A Comparison Between Genetic-Fuzzy and Neuro Fuzzy Systems (Seleção de Poços de Petróleo para Operação de Fraturamento Hidráulico: uma Abordagem Comparativa entre Sistemas Fuzzy-genético e Neuro-Fuzzy—In Portuguese). D.Sc. Thesis. Universidade Federal do Rio de Janeiro, Rio de Janeiro.

[15] Santos, J.A.C.M., Melo, R.C. and di Lullo, G. (2005) Case History Evaluation of RPMs on Conform Fracturing Applications. Proceedings of SPE Latin American and Caribbean Petroleum Engineering Conference (Society of Petroleum Engineers). http://dx.doi.org/10.2118/94352-MS

[16] Mahajan, M., Rauf, N., Gilmore, T. and Maylana, A. (2006) Water Control and Fracturing: A Reality. Proceedings of SPE Asia Pacific Oil \& Gas Conference and Exhibition (Society of Petroleum Engineers), Adelaide, 11-13 September 2006, 1-11. http://dx.doi.org/10.2118/101019-MS

[17] Santos, J.A.C.M., Cunha, R.A., Melo, R.C., Aboud, R.S., Pedrosa, H.A. and Marchi, F. (2009) Inverted-Convection Proppant Transport for Effective Conformance Fracturing. SPE Production Operations, 24, 187-193. http://dx.doi.org/10.2118/109585-PA 
Scientific Research Publishing (SCIRP) is one of the largest Open Access journal publishers. It is currently publishing more than 200 open access, online, peer-reviewed journals covering a wide range of academic disciplines. SCIRP serves the worldwide academic communities and contributes to the progress and application of science with its publication.

Other selected journals from SCIRP are listed as below. Submit your manuscript to us via either submit@scirp.org or Online Submission Portal.
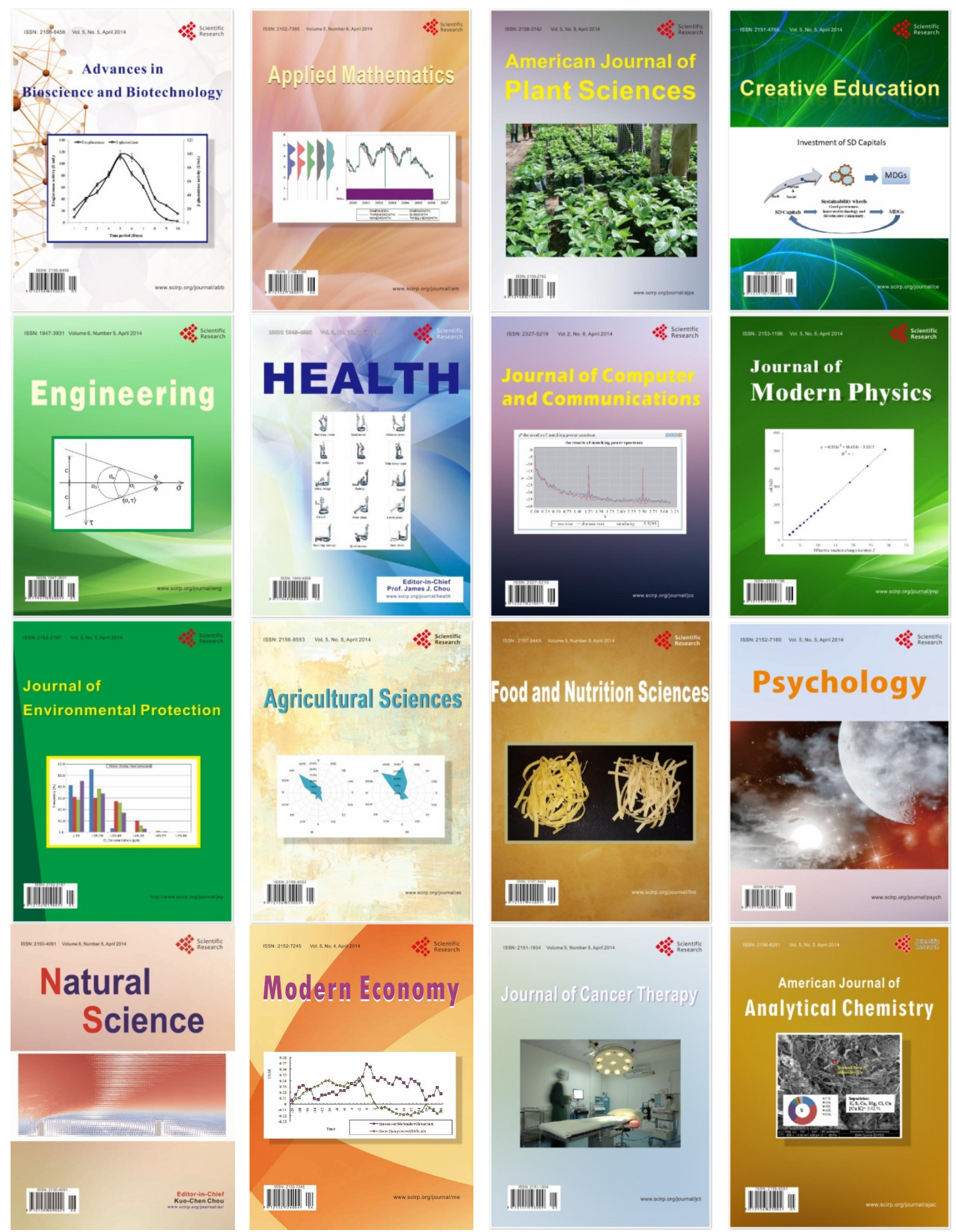